\title{
AUTOLOGOUS BLOOD TRANSFUSION: A SAFE STRATERGY FOR CONSERVING BLOOD
}

Ravindra S. Giri¹, Mukarram Iqbal2 ${ }^{2}$ Ajay G. M³

\section{HOW TO CITE THIS ARTICLE:}

Ravindra S. Giri, Mukarram Iqbal, Ajay G. M. "Autologous Blood Transfusion: A Safe Stratergy for Conserving Blood". Journal of Evolution of Medical and Dental Sciences 2015; Vol. 4, Issue 73, September 10;

Page: 12775-12782, DOI: $10.14260 /$ jemds/2015/1838

ABSTRACT: Autologous blood transfusion meaning "collection and reinfusion of the patient's own red blood cells" has been known to mankind since the time of Philip Physic of Philadelphia who in 1785 transfused the blood collected from post-partum bleeding patient. The first documented autologous transfusion was done by Blundell in 1818 by salvaging vaginal blood during post-partum hemorrhage. The technique employed was rather crude, it marked the beginning of the "Era of Autologous Blood transfusion". Autologous blood transfusion is justified for two reasons mainly, the prevention of allogeneic blood transfusion reactions and conservation of rare blood group types. There are four types of autologous transfusion. The pre deposit autologous donation, intra operative cell salvage, postoperative cell salvage, acute normovolaemic hemodilution. Each variety has advantages and disadvantages of its own but the issues which have to be borne in mind before adapting any of the technique is its feasibility, the cost effectiveness, fulfill the purpose of serving compatible blood without risking the patient's health status especially peri-operatively. India being one of the fastest developing nations of the world with whole lot of patient load and rather much larger deficit of compatible blood to patient should harvest health resources in improvising the technology and feasibility of autologous blood transfusion.

KEYWORDS: Pre deposit autologous transfusion intra/Post-operative cell salvage a cute normovolemic hemodilution pregnancy.

INTRODUCTION: Autologous blood transfusion meaning "collection and reinfusion of the patient's own red blood cells" has been known to mankind since the time of Philip Physic of Philadelphia who in 1785 transfused the blood collected from post-partum bleeding patient. The first documented autologous transfusion was done by Blundell in 1818 by salvaging vaginal blood during post-partum hemorrhage. The technique employed was rather crude, it marked the beginning of the "Era of Autologous Blood transfusion".

Definition: Blood collected from patient for re transfusion at later time into the same individual is called autologous blood transfusion.(1)

\section{Advantages:}

- Prevent transfusion transmitted diseases.

- Prevent red cell allo-immunization.

- Supplements the blood supply.

- Provides compatible blood for patients especially with rare blood groups.

- Prevent adverse transfusion reactions.

- Provide solution to religious belief (Jehovah's witness).

- Provides reassurance to patients concerned about blood risks.(2) 


\section{Disadvantages:}

- Risk of bacterial contamination is not reduced.

- Risk of ABO incompatibility error is not reduced.

- Is more costly than allogenic blood.

- Results in wastage of blood not transfused with allo antibodies.

- Increased incidence of adverse reactions to autologous donation.

- Subjects patients to peri operative anaemia and increased likelihood of transfusion.

- Donors of autologous blood are not considered as voluntary blood donors for allogenic transfusions, so the unused autologous blood cannot be used for allogenic transfusion.(2)

\section{Types of autologous transfusion;}

1. Pre deposit autologous donation (PAD).

2. Intra and post-operative cell salvage.

3. Acute normovolemic hemodilution (ANH).(2)

\section{A. Pre deposit autologous donation;}

- Pre deposit autologous donation is banking of red cell units from the patient before planned surgery.(3)

Advantages: Individuals planned to undergo elective surgical procedures are not exposed to blood of other persons.

\section{Disadvantages:}

- Time is needed for the collection prior to surgery. So this technique is not feasible for emergency surgical procedures.

- It is costlier and uses additional resources of a hospital.

- Patients may not be able to pre-donate all the blood they require for surgery. So again there is a slight risk of patient being exposed to allogenic transfusion

- Blood is discarded if not transfused back to the donor.

- There is an increased probability of the patent receiving a transfusion if their blood is available.(3)

Patients posted for elective surgical procedures may be offered autologous blood whenever collection and supply is clinically feasible. Points that should be taken into account include:

- Clinical indications supporting the potential need for blood transfusion.

- $\quad$ Selecting appropriate patients.

- Clinical supervision of autologous blood collection.

- The procedures for testing, storage, transport and supply of autologous blood.

- Ethical and legal implications of providing or not providing access to autologous blood supply.

\section{Consent:}

- The patient or person legally responsible for the patient should give their informed consent for the procedure. The consent should include:

- The risks and benefits associated with autologous blood collection. 
- That the range of tests performed will be in keeping with any current required blood transmissible disease markers for allogeneic blood and that any test result which is not negative for these markers will result in the units being destroyed.

- That other allogeneic blood or blood products may be needed.

- Permission to notify the patient's physician of tests for blood transmissible disease markers that are not negative.

- That blood will be discarded if not used by the patient.(3)

\section{Donation Criteria:}

- Patients considered for autologous blood collection must be assessed by the treating physician who must be satisfied that they are physically fit to have the required volume of blood removed.

- Caution should be exercised where a patient has a history of cardiovascular disease especially ischemic heart disease.eg. Angina and myocardial infarction.

- Patients should not have a history of recent cerebrovascular symptoms including transient ischaemic attacks or a stroke.

- Patients should not have severe hypertension. Medical review is required if the blood pressure is over systolic $180 \mathrm{~mm} \mathrm{Hg}$ or diastolic $100 \mathrm{~mm} \mathrm{Hg}$.

- Patients should not have severe respiratory disease with breathlessness at rest

- It is also recommended that patients who test not negative for any of the currently approved blood transmissible disease markers should be excluded from autologous blood collection

- There are no upper age limitations to autologous collection. Patients over the age of 65 should be considered in the context of their general health.

- There are no body weight limitations for patients undergoing autologous blood collections. Patients who weigh less than $50 \mathrm{~kg}$ should have $8 \mathrm{ml} / \mathrm{kg}$ collected.

- Estimation of the haemoglobin should be carried out before each blood collection and should be $11 \mathrm{~g} / \mathrm{dL}$ or greater. ${ }^{(3)}$

Contraindications: Patients with a range of medical conditions should not normally be considered for autologous collection. They include:

- Incompletely controlled epilepsy.

- A history of syncope after blood collection.

- Low blood pressure

- Low haemoglobin, generally set a minimum level of $11 \mathrm{~g} / \mathrm{dL}$ for females and males before each collection of blood. If it falls below this value the patient should be reviewed by the treating physician.

- Haematological conditions which will affect the storage of the red cells (e.g., Sickle Cell disease, unstable haemoglobinopathy etc.)

- Pregnancy is not an absolute contraindication, but collection of autologous blood from a pregnant woman would rarely be considered medically appropriate.

PAD in Pregnancy: Pre-delivery autologous blood deposit is not recommended. A Cochrane review on preoperative autologous donations 33 considered randomized controlled trials and concluded that the 
studies were of poor quality, with insufficient numbers of patients and high transfusion rates. None of the studies were conducted on pregnant women.(4)

\section{Blood Collection:}

- Collection and storage of blood for autologous transfusion will only be initiated upon the written authority of the clinician/surgeon requesting the units. Each collecting facility should have a standard referral and request form to be completed by the requesting clinician/surgeon.

- The patient must have a confirmed operation date.

- For a patient more than $50 \mathrm{~kg}$ a standard collection is $450 \mathrm{ml}$.

- Patients considered suitable for autologous blood collection can have 1-5 units of their blood stored.

- $\quad$ Collection may commence up to 28 days prior to surgery. Each unit can be stored for up to 35 days. Autologous units are usually collected at weekly intervals (minimum recommended interval is 72 hours), with the last collection at least 72 hours before surgery to permit equilibration of plasma proteins and restoration of blood volume.

- Patient must not be NPO for more than 3 hours prior to blood collection and should rest for 1530 minutes after the procedure.

- Oral fluid replacement is recommended following collection. The doctor supervising the autologous programme may recommend a patient not to participate or continue due to medical reasons. Patients are also free to withdraw from autologous blood collection programmes at any time.(3)

\section{Labelling of Collected Blood:}

- The label or the tag should contain following information.

- Name of the blood bank (collecting facility) and its manufacturing licence number.

- Name of the patient and the hospital where he is hospitalised.

- Patient's hospital registration number.

- $\quad$ ABO and Rh type.

- Date of collection and expiry.

- $\mathrm{HIV} / \mathrm{HCV} / \mathrm{HbSAg} / \mathrm{VDRL}$ status.

- Malarial parasite status.

- A Note saying "for autologous transfusion only".

\section{Laboratory Testing:}

- Each pre-deposited autologous unit must be tested for ABO and Rh D to confirm the patient's blood group.

- It is recommended that screening for currently approved blood transmissible disease markers for allogeneic blood be performed on autologous units. This testing of autologous units despite increased costs maximises safety.

- Units not negative for blood transmissible disease markers should be destroyed.

Storage: Pre deposit autologous blood should be stored in a refrigerator at a controlled temperature between $2-6^{\circ} \mathrm{C}$, with alarms set at $3-5^{\circ} \mathrm{C}$ and physically separated from allogeneic blood stocks and cross-matched blood. 
An unused unit of blood collected for autologous transfusion must not be transfused to another patient. Unused units of autologous blood should be kept until expiry and then be discarded.(3)

Acute normovolemic hemodilution (ANH): Haemodilution is defined as the artificial reduction of the erythrocyte count in the blood by dilution with crystalline or colloidal fluids. The treatment is aimed at reducing blood loss.

In acute normovolemic haemodilution (ANH), a number of units of blood are collected from a large vein, immediately pre-operatively after the patient is under anaesthesia, until the desired Hb has been reached. The blood is replaced volume for volume with a plasma substitute (normovolemia).(5)

For non-cardiac surgical procedures, ANH is used with the aim of lowering the number of circulating erythrocytes by dilution, resulting in a smaller net loss of erythrocytes. This also applies to cardiac surgical procedures, whilst also preventing the collected blood from being exposed to activation by the use of the cardiopulmonary bypass machine.

The Procedure: The quantity of blood that can be collected depends on the initial Haematocrit of the patient and the estimated blood volume. Usually employed for the procedures involving blood loss more than $20 \%$ of HCT.

The blood is usually collected in standard bags containing anticoagulants on a tilt rocker with automatic volume sensors, carried out usually by anaesthetists with close monitoring of patients circulating volume and perfusion status.

Withdrawal of blood should be accompanied by simultaneous infusion of either crystalloids ( $3 \mathrm{ml}$ of crystalloid for every $1 \mathrm{ml}$ of blood being drawn) or colloid ( $1 \mathrm{ml}$ of colloid for every $1 \mathrm{ml}$ of blood drawn).

The blood collected under sterile conditions can be stored at room temperature for a maximum of 6 hours and at $4{ }^{\circ} \mathrm{C}$ for 24 hours. The platelet function of the collected blood will be lost with storage at $4{ }^{\circ} \mathrm{C}$.

The blood collected will not be tested for transmissible microorganisms.

The collected blood doesn't leave operating room.

Return of the blood takes place in reverse order, because the unit that was collected first contains the highest number of erythrocytes, platelets and clotting factors ('last out, first in').(5,1)

Hemodynamic Consequences: Withdrawal of blood and replacement with crystalloids leads to reduction in the oxygen content of the arterial blood.

Compensatory hemodynamic mechanisms and surplus oxygen delivery capacity of the blood makes ANH safe.

Reduction in red cell number leads to decrease in the viscosity and peripheral resistance of the blood, thus increasing the cardiac output and oxygen delivery to the tissues indirectly.(2)

Safety of ANH: The reduction in viscosity causes vasodilation and increases cardiac output. It is recommended to monitor patients with cardiac conditions closely using ultrasound Doppler or via cardiac output monitoring. ANH can also result in an extension of the neuro-muscular block when using rocuronium, but not with cisatracurium.

Dilution can cause the concentration of clotting factors to decrease. The use of large quantities of plasma expanders can cause coagulopathy. 
A part of the infused plasma substitute or the protein solution used disappears into the "endothelial surface plasma layer" (Glycocalix) and another part leaves the circulation. This explains the fact that approximately $15 \%$ more plasma substitute is required to replace the collected volume of blood.

ANH is a cheap and easy technique to apply.(5)

Contraindications: The contraindications for the application of ANH are:

- Pre-operative anaemia.

- Sepsis.

- Heart failure or ischaemic heart disease.

- Myocardial infarction.

- Cardiogenic shock.

- Severe pulmonary disease.(5,6)

Intra and post-operative cell salvage (Peri-operative auto transfusion): Peri-operative autotransfusion is a type of autologous blood transfusion in which the blood lost peri-operatively is returned to the patient during or immediately after the operation, usually within 6hrs.

\section{Two types of Peri operative Cell Salvage:}

1. Unprocessed Cell Salvage: The blood is usually filtered, but re-infused without washing. It involves the re-infusion of drain blood. This can be done up to 6 hours post-operatively. The upper limit for such post-operative unprocessed auto-transfusion in adults is no more than $15 \%$ of the circulating blood volume, with a maximum of $1,500 \mathrm{~mL}$.

2. Processed Cell Salvage: Processed auto-transfusion is a method in which the peri-operatively collected blood is washed and separated by a machine. The final component consists of concentrated erythrocytes in $\mathrm{NaCl} 0.9 \%$. The method can be continued post-operatively for up to 6 hours after connection of the wound drain. Under certain conditions, the post-operative period can be extended to 18 hours. The temperature at which the blood is stored becomes important in that case.

\section{Indications:}

1. Massive blood loss of more than $>20 \%$ of the circulating blood volume.

2. Unavailability of compatible blood due to rare blood groups.

3. Blood collected in the abdominal or pelvic cavity due to organ rupture or any major vascular injury.

4. Jeehovaas witnesses.

\section{Contraindications:}

- Rinsing with toxic substances.

- Locally used haemostatics.

- Bacterial contamination (Relative)-Bacteria are not washed away completely. In emergency situations, antibiotics can be given in the case of bacterial contamination. 
- Tumour surgery (Relative) - Centrifugation and washing does not result in removal of all tumour cells, but irradiation of washed blood with 50Gy results in at least a $10 \log$ reduction of the number of viable tumour cells. A combination of leukocyte filtration followed by irradiation effectively disables active tumour cell.(7)

- Sickle cell anaemia.(5)

Procedure: The blood from the surgical drain is collected into the sterile canisters and subjected to filtration through membrane called leucocyte filter (Having a pore size of 40 microns ) and fat filter. Then the RBCs are subjected to washing process and later suspended in $0.9 \%$ saline. These washing process reduces the leucocyte load, bacterial load and also various inflammatory mediators thus reducing the incidence of various complications.

\section{Advantages:}

1. Oxygen carrying capacity of the recovered RBCs are equivalent to stored allogenic RBCs.

2. Adequate number of viable platelets would be available if transfused within 4-6hrs.

3. Blood can be collected first and one can decide at a later stage whether it should be processed and/or returned to the patient. This significantly reduces the costs.

4. The equipment is usually operated by anaesthesiology technician's or-in case of post-operative use-by recovery room nurses/ward nurses which does not require additional specialised personnel.

Disadvantages: The blood collected from surgical drain contains high concentration of lysed RBC, leucocytes, free hemoglobin, activated complement factors, cytokines, interleukin 6, bacteria which can predispose the patient to various complications such as:

1. ARDS.

2. DIC.

3. Renal function abnormalities,

4. Multi - organ failure ('blood salvage' syndrome),

5. Air or thromboembolism and,

6. Coagulopathies.

Role of Intra Operative Cell Salvage in Pregnancy: The routine use of cell salvage techniques in pregnancy is not recommended. But small case series have been conducted where in intraoperative cell salvage is done, however, large randomized control trials are yet to be conducted to prove its safety. The leucocyte filter used is efficient in removing amniotic fluid but not the fetal RBC, so cell salvage if done in Rh negative women, anti-D immunization (as determined by Kleihauer test 30-40 minutes after the procedure) will be required to prevent rhesus immunization in RhD-negative women.(4)

CONCLUSION: India being one of the fastest developing nations of the world with whole lot of patient load and rather much larger deficit of compatible blood to patients should harvest health resources in improvising the technology and feasibility of autologous blood transfusion. 


\section{BIBLIOGRAPHY:}

1. Norfolk D. Transfusion Medicine. 2005. 18-86 p.

2. Lawrence T. Goodnough and Terri G. Monk. Miller's Anesthesia. seventh. Miller RD, editor. CHURCHILL LIVINGSTONE ELSEVIER; 2010. 3075 p.

3. Collection AB. Topics in Transfusion Medicine. 2002;9(2).

4. Calkins L a. Blood Transfusion in Obstetrics and Gynecology*. South Med J. 1929;22(8):704-7.

5. Cbo. Blood Transfusion Guideline. ational Users' Board Blood Suply. 2011;

6. Boulton FE, James V, Stainsby D. Guidelines for policies on alternatives to allogeneic blood transfusion. 1. Predeposit autologous blood donation and transfusion. Transfus Med. 2007;17(5):354-65

7. Transfusion AS of B. Topics in Transfusion Medicine. Transfusion. 1996;3(2).

\section{AUTHORS:}

1. Ravindra S. Giri

2. Mukarram Iqbal

3. Ajay G. M.

\section{PARTICULARS OF CONTRIBUTORS:}

1. Associate Professor, Department of Anaesthesiology, Mahadevappa Rampure Medical College (MRMC), Gulbarga.

2. Assistant Professor, Department of Anaesthesiology, Mahadevappa Rampure Medical College (MRMC), Gulbarga.

FINANCIAL OR OTHER COMPETING INTERESTS: None
3. Post Graduate, Department of Anaesthesiology, Mahadevappa Rampure Medical College (MRMC), Gulbarga.

\section{NAME ADDRESS EMAIL ID OF THE CORRESPONDING AUTHOR:}

Dr. Ravindra S. Giri,

H/No: 2-909/67/49,

Swastik Nagar, Gulbarga-585105, Karnataka.

E-mail: ajaygm02@gmail.com

Date of Submission: 19/08/2015.

Date of Peer Review: 20/08/2015.

Date of Acceptance: 04/09/2015.

Date of Publishing: 09/09/2015. 